\title{
An Introduction to Neurotology : Anirban Biswas
}

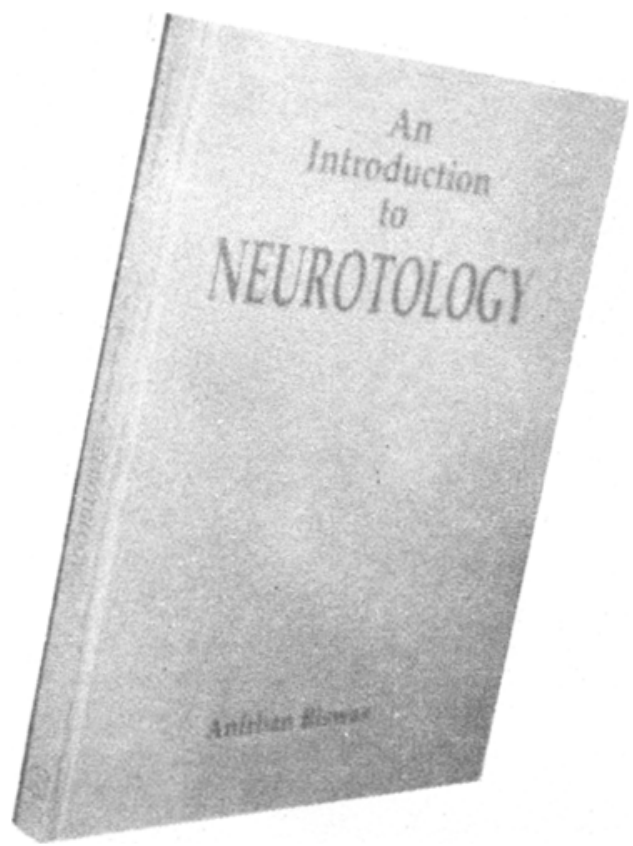

One can be happy with Dr. Anirban Biswas, who in the foreward to this book writes, "The purpose of writing this book is primarily to standardise the protocols for clinical neurotological examination to acquaint the clinician with the information available from the sophisticated neurotological investigations and also apprise him of the various treatment modalities available".

This book has really fulfilled the purpose of the author. However, addition of a chapter on paediatric otoneurology would have been a near total fulfilment which is found to be absent.

This 135 page book is published in A4 format with 69 figures of which 3 in colour.

The first 3 chapters deal with development, functional anatomy, history taking and clinical tests in neurotology. Chapter 4-6 with imaging and diagnosis work up, chapter 7 with neurotological disease, chapter 8-9 with conservative and surgical management of vestibular dysfunction. Chapter 10 with the analysis of neurotological cases which in effect is the meal of the book.

This is a well produced book with clear diagrams. But the standard of the X'Ray photographs are slightly disappointing. However, it shares the authors wealth of experience in the understanding and management of the problems associated with neurotological diseases and should be considered essential reading for students, teachers and practitioners in the field of E.N.T.

Santanu Banerjee

\section{Review Report of the North Eastern Branch of AOI News Bulletin Inaugural Issue, March 1998}

The North Eastern Branch of the Association of Otolaryngologists of India released the inaugural issue of their Quarterly News Bulletin in March 1998 under the able editorship of Dr. P. M. Baruah.

The highlights of the Bulletin included a Summary of the prestigious Dr. R.A.F. Cooper Award the 6th Guwahati Umiversity oration on 16th January 1998 International Research breakthrough in the field of "Smell" and the Hon. Secretary's message in brief. It also provided interesting information regarding various conferences and workshops held, guest lectures delivered and awards received by various eminent E.N.T. Consultants.

A brief summary with the highlights of the Golden Jubilee Conference of AOI held at New Delhi was aptly given with valuable information regarding all conferences and workshops to be held in 1998 with an update of the achievements of various eminent AOI members in 1997.

The News Bulletin covers briefly but precisely every event of the North Eastern Branch as well as important events of the Association of Otolaryngologists of India with all possible informative ENT news.

B. T. Hathiram 